\title{
Attractive double-layer forces between neutral hydrophobic and neutral hydrophilic surfaces
}

\author{
Eduardo R. A. Lima, Mathias Boström, Nadine Schwierz, \\ Bo Sernelius and Frederico W. Tavares
}

Linköping University Post Print

N.B.: When citing this work, cite the original article.

Original Publication:

Eduardo R. A. Lima, Mathias Boström, Nadine Schwierz, Bo Sernelius and Frederico W. Tavares, Attractive double-layer forces between neutral hydrophobic and neutral hydrophilic surfaces, 2011, Physical Review E. Statistical, Nonlinear, and Soft Matter Physics, (84), 6, 061903.

http://dx.doi.org/10.1103/PhysRevE.84.061903

Copyright: American Physical Society http://www.aps.org/

Postprint available at: Linköping University Electronic Press http://urn.kb.se/resolve?urn=urn:nbn:se:liu:diva-73312 


\title{
Attractive double-layer forces between neutral hydrophobic and neutral hydrophilic surfaces
}

\author{
Eduardo R. A. Lima, ${ }^{1,2, *}$ Mathias Boström, ${ }^{3}$ Nadine Schwierz, ${ }^{4}$ Bo E. Sernelius,,${ }^{3, *}$ and Frederico W. Tavares ${ }^{2,5}$ \\ ${ }^{1}$ Instituto de Química, Universidade do Estado do Rio de Janeiro, PHLC, CEP 20550-900 \\ ${ }^{2}$ Programa de Engenharia Química, COPPE, Universidade Federal do Rio de Janeiro, 21945-970, Rio de Janeiro, Rio de Janeiro, Brazil \\ ${ }^{3}$ Department of Physics, Division of Theory and Modeling, Chemistry and Biology, Linköping University, SE-581 83 Linköping, Sweden \\ ${ }^{4}$ Department of Physik, Technische Universität München, D-85748 Garching, Germany \\ ${ }^{5}$ Escola de Química, Universidade Federal do Rio de Janeiro, Cidade Universitária, CEP 21949-900, Rio de Janeiro, Rio de Janeiro, Brazil
}

(Received 6 September 2011; revised manuscript received 17 October 2011; published 6 December 2011)

\begin{abstract}
The interaction between surface patches of proteins with different surface properties has a vital role to play driving conformational changes in proteins in different salt solutions. We demonstrate the existence of ion-specific attractive double-layer forces between neutral hydrophobic and hydrophilic surfaces in the presence of certain salt solutions. This is performed by solving a generalized Poisson-Boltzmann equation for two unequal surfaces. In the calculations, we utilize parametrized ion-surface potentials and dielectric-constant profiles deduced from recent non-primitive-model molecular dynamics simulations that partially account for molecular structure and hydration effects.
\end{abstract}

DOI: 10.1103/PhysRevE.84.061903

PACS number(s): 87.16.A-, 82.70.Dd, 34.20.Gj

\section{INTRODUCTION}

In this paper, we explore a previously unrecognized contribution to the attraction between an uncharged hydrophobic and an uncharged hydrophilic surface. Figure 1 presents a schematic of such hydrophobic and hydrophilic surfaces interacting across a salt solution. Ion-specific double layers of opposite signs may be set up at the two surfaces leading to an attractive force. We propose that this may be important to account for when considering the interaction between surface patches of different proteins having different surface properties [1]. Such an effect was predicted by Schwierz et al.. based on their calculated double layers that had opposite signs at a single hydrophobic surface as compared to a single hydrophilic surface [2]. Our approach is to solve a generalized PoissonBoltzmann (PB) equation [3] for two unequal planar surfaces. In this solution, water is treated as a continuum. However, we partially account for molecular structure $[2,4]$, as well as dispersion potentials [5] and hydration effects [6] by utilizing ion-surface parametrized potentials of mean force (PMF) and dielectric constant profiles deduced from very recent nonprimitive-model molecular dynamics (MD) simulations [2].

We show that the ion-specific double-layer force is similar in magnitude to the attractive Hamaker-van der Waals force at moderate to high salt concentrations (around 0.1-1 M). It clearly gives an important contribution to the short-range attraction. We note that previous papers on specific ion effects using the PMF from simulations have dealt with a single surface or with the interaction between two equal surfaces. This is a paper that addresses the ion-specific double-layer interaction between a hydrophobic and a hydrophilic surface in the presence of different salt solutions.

The structure of this paper is as follows. In Sec. II, we briefly present the basic theory of the generalized PB equation and include a very short recapitulation of the parametrized PMFs used. We also give the asymptotic expression for the

\footnotetext{
${ }^{*}$ Corresponding authors.
}

bos@ifm.liu.se

(BES);
Hamaker-van der Waals force between two self-assembled monolayers (SAMs) that is used as a comparison with the double-layer pressure; the expression is appropriate when the surfaces are not so close that they overlap but at the same time not so far apart that the finite thickness of the SAM becomes relevant. Then, in Sec. III, we present the results of our calculations for two unequal surfaces. These results demonstrate that attractive double-layer forces are set up between an uncharged hydrophobic and an uncharged hydrophilic surface in the presence of certain salt solutions. Finally, Sec. IV, we end with some concluding remarks.

\section{THEORY}

In the classical Derjaguin, Landau, Verwey, and Overbeek (DLVO) theory, van der Waals-Lifshitz forces are treated separately in a linear inconsistent way [5]. In such a standard theory, the PB equation only takes the electrostatic potential into account. After the solution is obtained, the direct van der Waals interaction between the two planar surfaces is added. This ansatz is incomplete, and the theory cannot predict ion specificity, which is commonly observed experimentally [5]. In a more complete theory, additional nonelectrostatic (NES) interactions [5-8] and the effect of short-range hydration [6] must be treated at the same level as the electrostatic forces acting on the ions.

Extending earlier papers that considered two equal surfaces [9], we use an ion-specific generalized PB equation for two unequal neutral surfaces a distance $L$ apart,

$$
\begin{gathered}
\varepsilon_{0} \frac{d}{d x}\left[\varepsilon(x) \frac{d \psi}{d x}\right] \\
=-e \sum_{i} c_{0, i} z_{i} \exp \left\{-\left[z_{i} e \psi+U_{i}(x)\right] / k_{B} T\right\}, \\
\left.\frac{d \psi}{d x}\right|_{x=0}=0,\left.\quad \frac{d \psi}{d x}\right|_{x=L}=0,
\end{gathered}
$$

where $k_{B}$ is the Boltzmann constant, $z_{i}$ is the valency of the ions, $e$ is the elementary charge, $T$ is the temperature of the system, $\psi$ is the self consistent electrostatic potential, and 

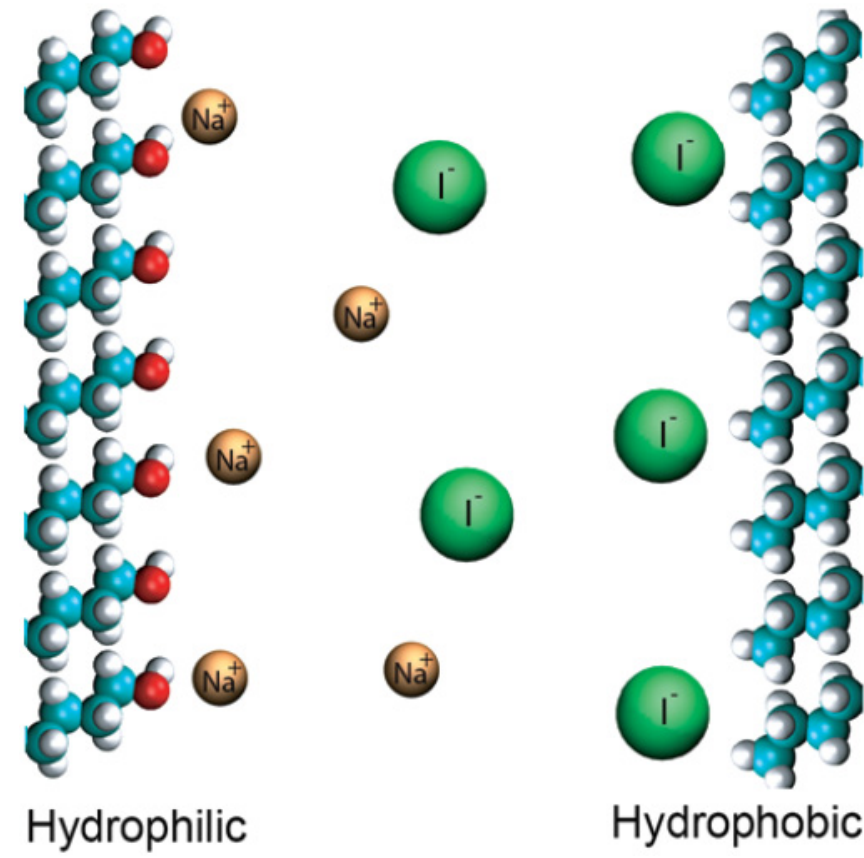

FIG. 1. (Color online) A schematic of hydrophobic and hydrophilic surfaces interacting across a salt solution. Opposite signs of the double layers can give rise to ion-specific attraction.

$U_{i}(x)=W^{a}(x)+W^{b}(L-x)$ is the sum of the ionic potentials (PMF) acting between the ion and each of the two surfaces (surface $a$ is hydrophobic and surface $b$ is hydrophilic). Schwierz et al. . performed simulations that gave the PMF for ions and $\varepsilon(x)$ shown in Figs. 2 and 3 near a single surface [2]. The local dielectric constant $\varepsilon(x)$ is defined as a function of position $x$ from the hydrophobic surface. Following our previous paper, we define the local dielectric constant $\varepsilon(x)$ as a function of position $x$ based on density simulation results, considering that these two quantities are highly correlated [2,9]. For the interaction between two surfaces, an important

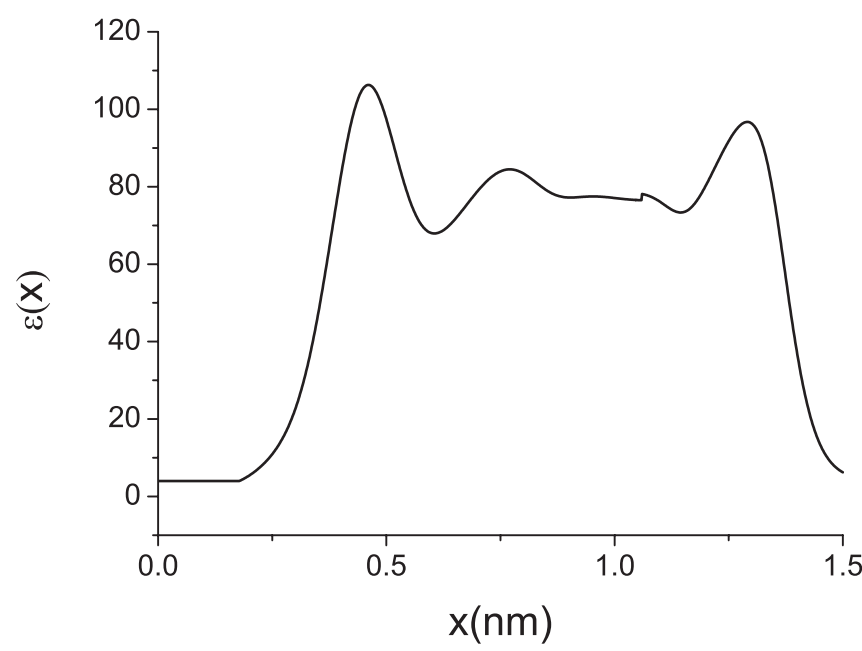

FIG. 2. Local dielectric constant as a function of the position for a surface-to-surface distance $L=1.5 \mathrm{~nm}$. The hydrophobic surface is located at $x=0$, and the hydrophilic surface is located at $x=$ $1.5 \mathrm{~nm}$.

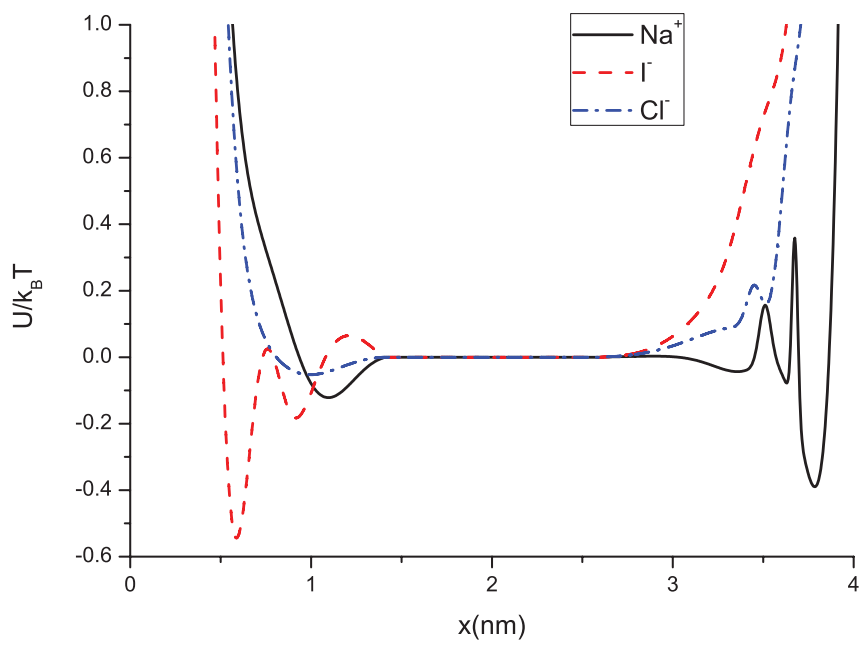

FIG. 3. (Color online) Parametrized PMFs between a hydrophobic surface at $x=0 \mathrm{~nm}$ and a hydrophilic surface at $x=4 \mathrm{~nm}$ for different ions (using, in all examples, parameters given in the supporting material of Ref. [2]).

issue is how to treat the overlap of the dielectric functions of both surfaces for short distances between the plates. Because of that, we do not consider any calculation for separating distances $(L)$ shorter than $1.5 \mathrm{~nm}$. For distances between 1.5 and $3 \mathrm{~nm}$ (where there is overlap), we carefully choose an adequate point of intersection in order to not take away important parts of these curves. In Fig. 2, in order to clarify this question, we show the local dielectric constant as a function of position $(x)$ between the surfaces for $L=1.5 \mathrm{~nm}$ where there is an overlap of the original dielectric functions of the single plates. In this case, we chose $x=1 \mathrm{~nm}$ as the connection point. One can notice that, in this point, there is a very small discontinuity that does not affect the numerical results presented in other figures. We base our paper on realistic NES potentials based on parametrized PMFs and dielectric profiles obtained by MD simulations of ions in solution near an uncharged hydrophobic or an uncharged hydrophilic surface [2]. PMFs for three different ions between these two surfaces are reproduced in Fig. 3 [2]. We observe that the cations $\left(\mathrm{Na}^{+}\right)$ are attracted toward the hydrophilic surface while anions (at least, $\mathrm{I}^{-}$) are attracted toward the hydrophobic surface. This leads to opposite signs of ion-specific double layers formed between the two different surfaces.

The electrostatic potential profiles and ion distributions obtained from the solution of Eq. (1) are used to calculate the pressure between the surfaces. The double-layer pressure between two planar surfaces at a distance $L$ can be calculated by the differentiation of the free energy of the system [9],

$$
P=-\frac{\partial}{\partial L}\left(\frac{A}{\text { area }}\right),
$$

and the free energy per unit of area is expressed by [9]

$$
\begin{aligned}
\frac{A}{\text { area }}= & \frac{e}{2} \int_{0}^{L} \psi \sum_{i} c_{i} z_{i} d x+\int_{0}^{L} \sum_{i} c_{i} U_{i} d x \\
& +k_{B} T \int_{0}^{L} \sum_{i} c_{i}\left[\ln \left(\frac{c_{i}}{c_{0, i}}\right)-1\right] d x .
\end{aligned}
$$


The first two terms on the right hand side of Eq. (4) are the energy contributions (electrostatic and the ionic potential of the MF contribution, respectively) to the free energy of the system, and the third term is the entropic contribution.

At large surface-to-surface distances where the ion-surface interaction potential approaches zero, Eq. (1) can be linearized corresponding to Debye-Hückel theory.

The solution of the linearized equation is

$$
\psi(x)=\psi_{\mathrm{DH}} \exp (-\kappa x)
$$

where $\kappa$ is the inverse Debye screening length $\kappa=$ $\sqrt{\left(e^{2} \sum_{i} c_{0, i} z_{i}^{2}\right) / \varepsilon \varepsilon_{0} k_{B} T}$. The resulting Debye-Hückel surface potential $\psi_{\mathrm{DH}}$ is a measure of the effective surface charge density $\sigma_{\mathrm{DH}}$,

$$
\sigma_{\mathrm{DH}}=\varepsilon \varepsilon_{0} \kappa \psi_{\mathrm{DH}}
$$

The term Debye-Hückel surface potential is a little misleading since this may lead the reader to think that the potential $\psi_{\mathrm{DH}}$ is the potential that one would find from the Debye-Hückel theory of the double layer. In fact, the potential $\psi_{\text {DH }}$ results from ion-specific effects at a hydrophobic surface or at a hydrophilic surface [2] and not from the standard Debye-Hückel theory. All ion-specific effects are included in the term $\psi_{\mathrm{DH}}$ and are assumed to be relevant only right at the surface. At all other distances, the far-field Debye-Hückel solution for the potential [Eq. (5)] is used.

The effective surface charge densities allow one to predict the long-range forces between two surfaces. At large surface separations where the exponentially decaying electrostatic potential is small, the pressure between the two surfaces can be calculated from [10]

$$
P(L)=\frac{\left(2 \sigma_{\mathrm{DH}}^{1} \sigma_{\mathrm{DH}}^{2}\right)}{\varepsilon \varepsilon_{0}} \frac{\left(2+e^{L \kappa}+e^{-L \kappa}\right)}{\left(e^{L \kappa}-e^{-L \kappa}\right)^{2}},
$$

where $\sigma_{\mathrm{DH}}^{i}$ is the effective surface charge density of the two surfaces, which is calculated from the Debye-Hückel surface potential [2]. Here, we use the bare dielectric constant of bulk water since the point of interest is the midplane between two remote surfaces.

The van der Waals-Hamaker pressure between two planar surfaces coated with SAMs is (at short range) approximately given by [11]

$$
P(L)=-\frac{A_{12}}{6 \pi L^{3}},
$$

where $A_{12}$ is the Hamaker constant between two selfassembled monolayers. Here, we use $A_{12}=1.21 k_{B} T$ based on the value reported by Kokkoli and Zukoski [12] for two SAMs of hexadecanethiol.

We calculate the Hamaker contribution to the pressure just to compare its magnitude to the double-layer pressure obtained by the modified PB theory. The van der Waals force always is present and plays an essential role in all phenomena involving intermolecular forces. Hence, the van der Waals force is a good reference function that can be used to check whether the magnitude of any other contribution is significant or not.

\section{RESULTS}

In Fig. 4(a), we explore the difference between the concentrations of cations and anions for $0.1 \mathrm{M} \mathrm{NaI}$ and $0.1 \mathrm{M}$ $\mathrm{NaCl}$. This quantity is proportional to the charge density profile between the plates. Ion-specific double layers of opposite signs are set up at the two surfaces in the presence of NaI. We show the corresponding curves for different concentrations of $\mathrm{NaI}$ in Fig. 4(b). This would suggest the possibility of an attractive pressure between these surfaces that increases with increasing salt concentration. The reader is referred to the cited paper (and the supporting materials of that paper) for simulation details [2].

In Fig. 5, we consider how the pressure between an uncharged hydrophilic and an uncharged hydrophobic surface depends on the specific choice of salt ions in the $1 \mathrm{M}$ salt solution used [ $\mathrm{NaCl}$ (dashed-dotted line) and $\mathrm{NaI}$ (solid line)]. At moderate to high salt concentrations, the effect is similar in
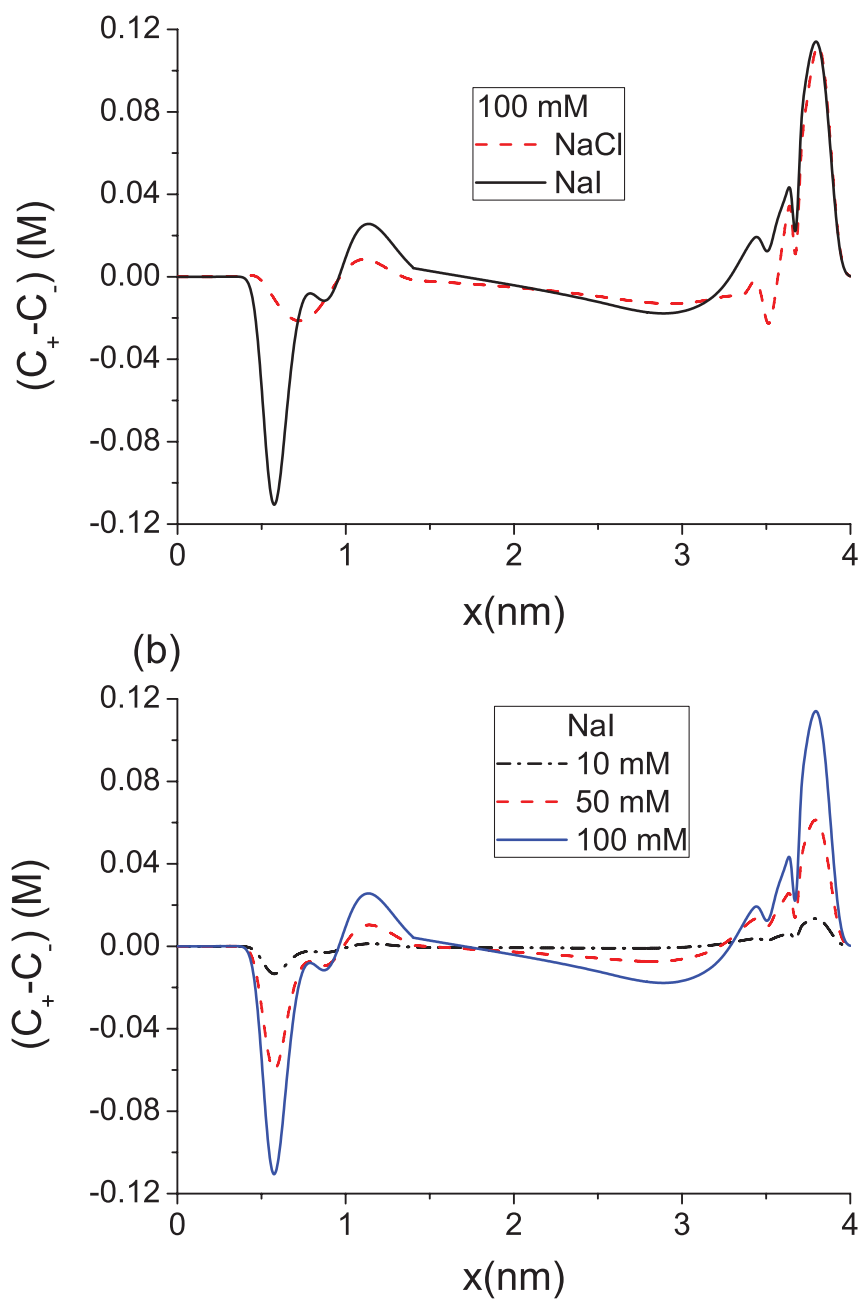

FIG. 4. (Color online) Difference between the concentration of cations $\left(C_{+}\right)$and anions $\left(C_{-}\right)$(proportional to the charge density profile) (a) for two different salt solutions: $0.1 \mathrm{M} \mathrm{NaCl}$ (dashed line) and $0.1 \mathrm{M} \mathrm{NaI}$ (solid line); (b) NaI at different concentrations: 0.01 $\mathrm{M}$ (dashed-dotted line), 0.05 M (dashed line), and 0.1 M (solid line) between an uncharged hydrophobic surface (left) and an uncharged hydrophilic surface (right) $4 \mathrm{~nm}$ apart. 


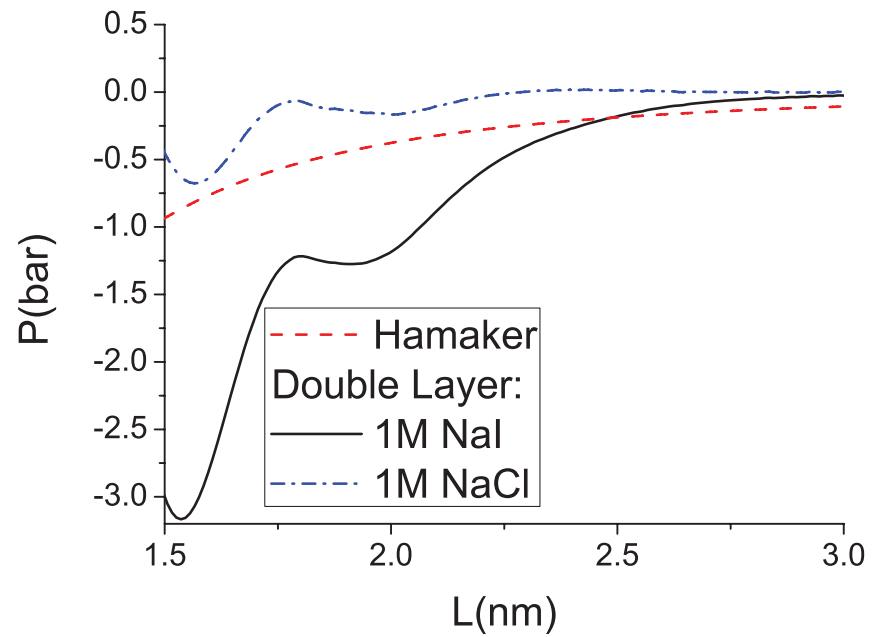

FIG. 5. (Color online) Van der Waals-Hamaker (dashed line) and double-layer pressure as functions of separation $L$ between an uncharged hydrophilic and a hydrophobic surface for $1 \mathrm{M} \mathrm{NaI}$ (full line) and $1 \mathrm{M} \mathrm{NaCl}$ (dash-dotted line) salt solutions.

magnitude to the attractive van der Waals-Hamaker pressure between the two surfaces (dashed line). We observe that the attraction between the two asymmetric surfaces increases with increasing size (and polarizability) of the anions. The doublelayer contribution is important and highly ion specific with stronger attraction for NaI (around four times the Hamaker attraction between the surfaces) and a weaker attraction for $\mathrm{NaCl}$ (smaller than the Hamaker interaction).

In Fig. 6, we show the comparison between the pressure calculated from PB theory and the pressure calculated from the effective surface charge densities using Eq. (7) as a function of the separation between the plates for the 1-M salt concentration. The agreement is perfect for long distances even for this high concentration. At the two different interfaces, ion-specific adsorption leads to effectively charged surfaces, which interact via screened Coulomb interactions with each other. In NaI

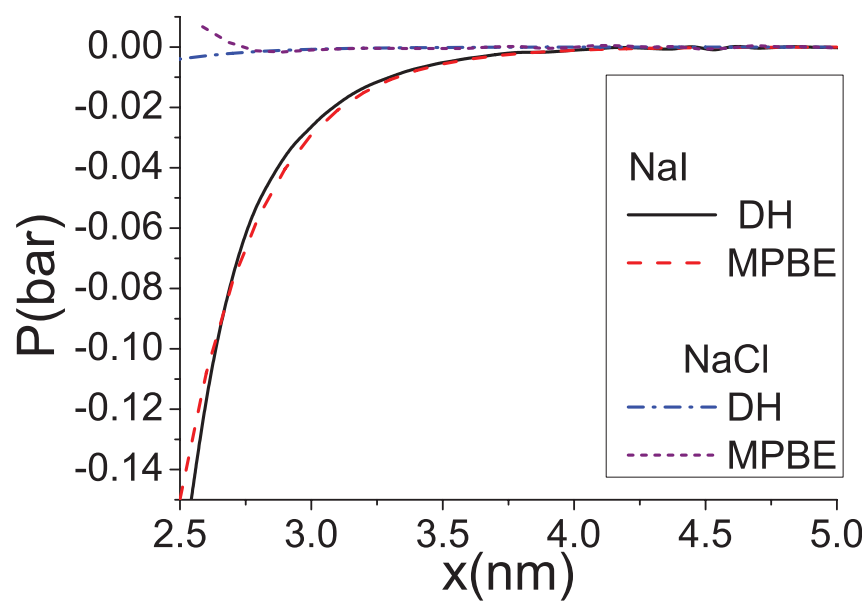

FIG. 6. (Color online) Comparison between the double-layer pressure calculated from Eq. (7) in the Debye-Hückel limit and the corresponding results obtained from the numerical solution of the modified PB equation for both salts at $1 \mathrm{M}$. $L$ is the separation between the surfaces.

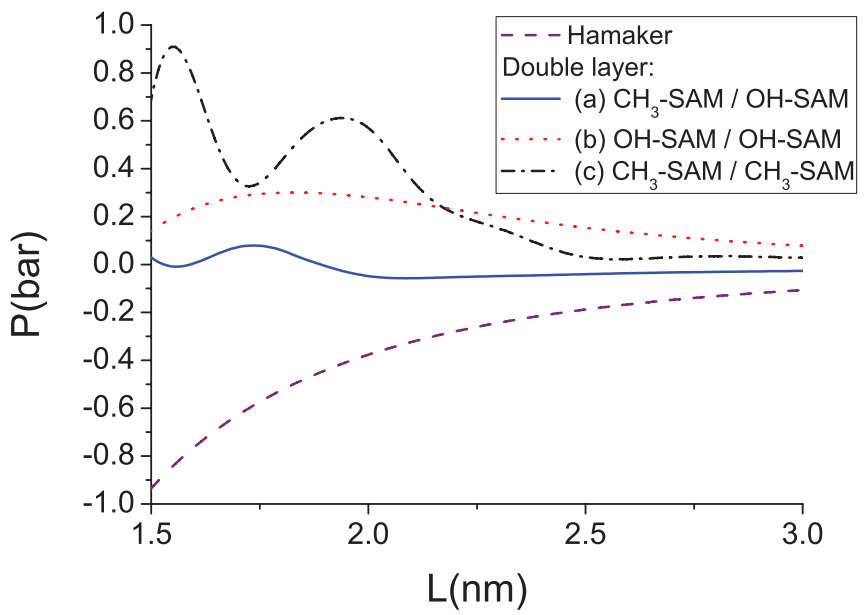

FIG. 7. (Color online) Van der Waals-Hamaker (dashed line) and double-layer pressure in $0.1 \mathrm{M} \mathrm{NaI}$ between (a) an uncharged hydrophilic and a hydrophobic surface (full line), (b) two uncharged hydrophilic surfaces (dotted line), and (c) two hydrophobic surfaces (dashed-dotted line). $L$ is the separation between the surfaces.

solutions, the long-range potential and, therefore, the effective surface charge density is negative at the hydrophobic surface but is positive at the hydrophilic surface [2]. This results in a long-range attraction and, therefore, a destabilization of uncharged pairs of hydrophobic-hydrophilic surfaces in $\mathrm{NaI}$ solutions. For $\mathrm{NaCl}$ solutions, a long-range attraction is predicted as well, which is much smaller than for $\mathrm{NaI}$ due to the smaller effective surface charges on both surfaces.

The question raised by our paper is if the double-layer pressure between different combinations of hydrophobic and hydrophilic SAMs are comparable in magnitude to the van der Waals-Hamaker pressure for $0.1 \mathrm{M}$ salt solutions. Since the pressures are higher for $\mathrm{NaI}$, we only present the result for this salt solution. In Fig. 7, we see that the double-layer pressure at the 0.1-M salt concentration is smaller in magnitude as compared to the double-layer pressure at the $1 \mathrm{M}$ salt concentration (Fig. 5). This is consistent with Fig. 4, which demonstrates that the double layers get more pronounced with increasing salt concentration. There also is an increasing screening effect with increasing salt concentrations, but this seems to be less important. We see that the repulsive double-layer pressure between two hydrophobic surfaces is similar in magnitude to the van der Waals-Hamaker pressure regardless of plate separation. However, the pressure between one hydrophobic surface and one hydrophilic surface is weakly repulsive at shorter distances and then, weakly attractive at longer distances. The double-layer repulsion between two hydrophilic surfaces is similar in magnitude to the Hamaker force for distances longer than $2 \mathrm{~nm}$.

In Fig. 8, we consider a $0.5 \mathrm{M}$ salt solution of $\mathrm{NaI}$. We see that, for this moderate salt concentration, there is strong double-layer repulsion between two hydrophobic surfaces due to large adsorption of iodide on both surfaces. For two hydrophilic surfaces, there is weak long-range repulsion and a weak short-range attraction. More importantly, at this concentration, we observe an attraction between one hydrophobic and one hydrophilic surface, which is on the 


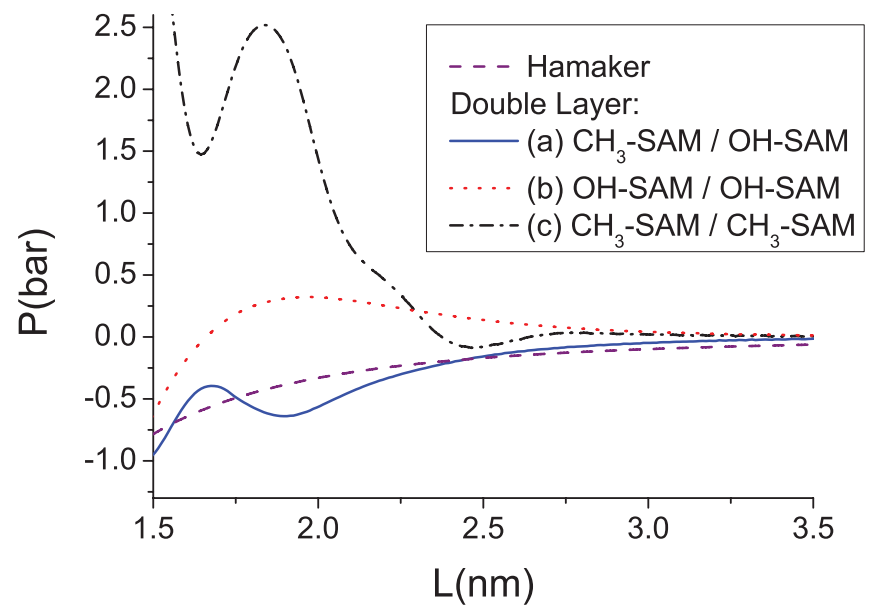

FIG. 8. (Color online) Same as Fig. 7, but for a $0.5 \mathrm{M}$ salt solution.

same order of magnitude as the Hamaker attraction regardless of the separation distance.

\section{CONCLUSIONS}

The electrostatics of charged particle adsorption (e.g., protein) to surfaces $[13,14]$ and interaction of dissimilar charged surfaces [15] have been explored in the past. However, our paper addresses the ion-specific double-layer attraction between an uncharged hydrophobic surface and an uncharged hydrophilic surface. Due to opposite signs of the double layers, the ion-specific double-layer pressure between an uncharged hydrophilic surface and an uncharged hydrophobic surface can give a strong attraction that is similar in magnitude to the Hamaker-van der Waals pressure. It is of vital importance to take this effect into account at moderate to medium salt concentrations (0.1-1 M) where, on one hand, the charge due to specific ion adsorption is not too small, and on the other hand, the screening length is not too short. We have found an ion-specific double-layer attraction between unequal surfaces. The attraction between the two asymmetric surfaces increases with increasing size (and polarizability) of the ions. This attraction is bound to be important for the interaction between patchy proteins with hydrophobic and hydrophilic surface patches [1].

\section{ACKNOWLEDGMENTS}

E.R.A.L. and F.W.T. acknowledge financial support from the FAPERJ, CAPES, and CNPq (Brazilian Agencies). M.B. and B.E.S. acknowledge financial support from the VRContract No. 70529001. We thank Professor Barry Ninham, Professor Roland Netz, Dr. Drew Parsons, and Dr. Dominik Horinek for valuable discussions.
[1] M. Lund and P. Jungwirth, J. Phys.: Condens. Matter 20, 494218 (2008).

[2] N. Schwierz, D. Horinek, and R. R. Netz, Langmuir 26, 7370 (2010).

[3] G. Luo, S. Malkova, J. Yoon, D. G. Schultz, B. Lin, M. Meron, I. Benjamin, P. Vanýsek, and M. L. Schlossman, Science 311, 216 (2006).

[4] D. Horinek and R. R. Netz, Phys. Rev. Lett. 99, 226104 (2007).

[5] B. W. Ninham and V. Yaminsky, Langmuir 13, 2097 (1997).

[6] D. F. Parsons and B. W. Ninham, Langmuir 26, 6430 (2010).

[7] M. Boström, D. R. M. Williams, and B. W. Ninham, Phys. Rev. Lett. 87, 168103 (2001).

[8] E. Wernersson and R. Kjellander, J. Chem. Phys. 125, 154702 (2006).
[9] E. R. A. Lima, D. Horinek, R. R. Netz, E. C. Biscaia, F. W. Tavares, W. Kunz, and M. Boström, J. Phys. Chem. B 112, 1580 (2008).

[10] V. A. Parsegian and D. Gingell, Biophys. J. 12, 1192 (1972).

[11] V. A. Parsegian, Van der Waals Forces: A Handbook for Biologists, Chemists, Engineers, and Physicists (Cambridge University Press, New York, 2005).

[12] E. Kokkoli and C. F. Zukoski, J. Colloid Interface Sci. 209, 60 (1999).

[13] P. M. Biesheuvel, M. van der Veen, and W. Norde, J. Phys. Chem. B 109, 4172 (2005).

[14] P. M. Biesheuvel, P. Stroeve, and P. A. Barneveld, J. Phys. Chem. B 108, 17660 (2004).

[15] P. M. Biesheuvel, J. Colloid Interface Sci. 275, 514 (2004). 